\title{
Heart rate response to Valsalva manoeuvre Reproducibility in normals, and relation to variation in resting heart rate in diabetics
}

\author{
V. S. BALDWA ${ }^{1}$ AND D. J. EWING \\ From the University Department of Medicine, Royal Infirmary, Edinburgh
}

The reproducibility of the heart rate response to the Valsalva manoeuvre was assessed in 12 young normal subjects who each repeated the manoeuvre 10 times. The length of the RR intervals before, during, and after each Valsalva manoeuvre was measured from an electrocardiogram and the changes expressed as two ratios: the 'tachycardia' ratio and the 'Valsalva' ratio. The within-subject variation was \pm 0.044 for the tachycardia ratio and $\pm 0 \cdot 18$ for the Valsalva ratio, while the between-subject variation was $0 \cdot 10$ for the tachycardia ratio and 0.37 for the Valsalva ratio. The heart rate response to the Valsalva manoeuvre was also found to be reproducible in 7 older normal subjects.

In 100 diabetic subjects with varying degrees of autonomic neuropathy, the heart rate response to the Valsalva manoeuvre was compared with the heart rate variation from a resting electrocardiogram. The heart rate variation correlated significantly with both the tachycardia ratio $(r=0.727, P<0.001)$ and the Valsalva ratio ( $r=0.659, P<0.001)$.

Thus, the heart rate response to the Valsalva manoeuvre is reproducible in normal subjects, and in diabetics, heart rate variation can simply be used as a test of autonomic function when more sophisticated tests are not available.

The Valsalva manoeuvre, named after Antonio Maria Valsalva (1666-1723), is widely regarded as a simple method of evaluating baroreflex integrity. Despite its use in many clinical and experimental situations, only limited studies of its reproducibility have been carried out (Levin, 1966). Changes in intra-arterial blood pressure have been used to assess the response to the Valsalva manoeuvre, but heart rate changes have also been shown to be reliable (Elisberg, 1963) and have the advantage of being noninvasive.

A further test of autonomic function has recently been proposed by Wheeler and Watkins (1973) in which the variation in resting heart rate was measured with a heart rate meter. The aim of the present study was first to define the reproducibility of the heart rate response to the Valsalva manoeuvre in normal subjects and secondly to see whether, in a group of patients with diabetes, measurements from a resting electrocardiogram of heart rate variation could reliably be used as a test of autonomic function.

'Dr. Baldwa held a post-doctoral Commonwealth fellowship in cardiorespiratory diseases. Present address: Reader in Medicine, S.M.S. Medical College and Hospital, Jaipur, India.

Received for publication 13 September 1976

\section{Subjects and methods}

Three groups of subjects were studied: the first group consisted of 12 normal, fit young subjects ( 8 men and 4 women, aged 20 to 41 years); the second 7 normal older subjects ( 3 men and 4 women, aged 52 to 66 years); and the third 100 diabetics, some of whom had definite autonomic neuropathy, some had impotence alone, while the remainder had no symptoms referable to the autonomic nervous system.

In each young normal subject the Valsalva manoeuvre was repeated at different times of the day on 10 occasions over a 2-month period. In each older normal subject the Valsalva manoeuvre was repeated 5 times over a one-week period. The 100 diabetics performed the manoeuvre on one occasion only.

Each subject performed a Valsalva manoeuvre by blowing through a mouthpiece attached to a manometer and maintaining a pressure of $40 \mathrm{mmHg}$ for 15 seconds. Care was taken that the subject was not blowing with his cheeks, and had an open glottis (a true Valsalva was clinically confirmed by a florid face and distension of the neck veins, and by a characteristic 'gasp' on release). Once the technique 
had been mastered the test was performed 3 times and the mean values used in calculating the results. Heart rate was measured from an electrocardiogram recorded continuously from one minute before until one minute after each manoeuvre. The subjects remained seated throughout and rested for at least 2 minutes between each Valsalva manoeuvre.

The longest and shortest $R R$ intervals were measured from the electrocardiogram before, during, and after the Valsalva manoeuvre and the following calculations were made.

(1) Mean resting heart rate (mean of longest and shortest RR interval before the manoeuvre).

(2) Variation in resting heart rate (difference between longest and shortest $R R$ interval before the manoeuvre).

(3) Tachycardia ratio (shortest RR interval during the manoeuvre divided by the longest $R R$ interval before).

(4) Valsalva ratio (longest $R R$ interval after the manoeuvre, divided by the shortest $R R$ interval during it).

\section{Results}

REPRODUCIBILITY IN YOUNG

NORMAL SUBJECTS

Table 1 summarises the findings in the young normal subjects. There were no statistically significant differences between the grouped data of the males and females: all the results have therefore been analysed together. Within-subject and between-subject variation was calculated by standard analysis of variance (Armitage, 1971). The withinsubject variation for the group (expressed as the standard deviation of repeated measurements within each individual) was 0.044 for the tachycardia ratio and 0.18 for the Valsalva ratio. The betweensubject variation for the group (expressed as the standard deviation of the mean values of all individuals) was $0 \cdot 10$ for the tachycardia ratio and 0.37 for the Valsalva ratio.
Interpretation of differences in successive observations in the same subject is often difficult in clinical practice. For normal individuals more than 95 per cent of successive readings would differ by less than 0.12 for the tachycardia ratio, and 0.51 for the Valsalva ratio. This is calculated from the formula $2 \times \sqrt{ } 2 \times S D$, since the variance of differences in successive observations is twice the variance of individual observations (Armitage, 1971).

\section{REPRODUCIBILITY IN OLDER}

NORMAL SUBJECTS

Table 2 shows the results in the older normal subjects. The mean resting heart rate and the mean tachycardia ratio in the older group were similar to the younger group, but the mean individual variation in resting heart rate and the mean Valsalva ratio were both lower in the older group, the differences just reaching statistical significance $(P<0.05)$. There were no statistically significant differences between the younger and older groups in their withinsubject variation $(0.043$ for the tachycardia ratio and 0.17 for the Valsalva ratio). The betweensubject variation was 0.05 for the tachycardia ratio and 0.18 for the Valsalva ratio.

\section{COMPARISON OF VALSALVA MANOEUVRE} WITH VARIATION IN RESTING HEART RATE IN DIABETICS

The tachycardia ratio and the Valsalva ratio in the 100 diabetics are shown in Fig. 1. Fig. 2 and 3 show the relation between the individual variation in resting heart rate and the tachycardia ratio (Fig. 2) and the Valsalva ratio (Fig. 3). After the influence of both age and the resting heart rate had been eliminated, using a partial correlation coefficient technique, there was close relation between the variation in resting heart rate and both the tachycardia ratio $(r=0.727, P<0.001)$ and the Valsalva ratio $(r=0.659, P<0.001)$ in the group as a whole.

Table 1 Heart rate responses to the Valsalva manoeuvre in 12 young normal subjects

\begin{tabular}{|c|c|c|c|c|c|c|}
\hline Subject & Age (y) & Sex & Resting RR interval (ms) & Variation in resting $R R$ interval (ms) & Tachycardia ratio* & Valsalva ratio * \\
\hline $\begin{array}{r}1 \\
2 \\
3 \\
4 \\
5 \\
6 \\
7 \\
8 \\
9 \\
10 \\
11 \\
12\end{array}$ & $\begin{array}{l}23 \\
34 \\
31 \\
22 \\
41 \\
30 \\
29 \\
28 \\
22 \\
32 \\
20 \\
22\end{array}$ & $\begin{array}{l}M \\
M \\
M \\
M \\
M \\
M \\
M \\
M \\
F \\
F \\
F \\
F\end{array}$ & $\begin{array}{l}733 \pm 77 \\
781 \pm 23 \\
831 \pm 44 \\
855 \pm 81 \\
736 \pm 64 \\
775 \pm 83 \\
990 \pm 58 \\
681 \pm 51 \\
724 \pm 41 \\
746 \pm 54 \\
592 \pm 42 \\
850 \pm 58\end{array}$ & $\begin{aligned} 136 & \pm 59 \\
117 & \pm 38 \\
77 & \pm 15 \\
161 & \pm 53 \\
133 & \pm 66 \\
193 & \pm 60 \\
277 & \pm 105 \\
51 & \pm 17 \\
124 & \pm 51 \\
87 & \pm 59 \\
91 & \pm 29 \\
220 & \pm 36\end{aligned}$ & $\begin{array}{l}0.61 \pm 0.05 \\
0.68 \pm 0.03 \\
0.73 \pm 0.03 \\
0.60 \pm 0.05 \\
0.57 \pm 0.03 \\
0.90 \pm 0.04 \\
0.60 \pm 0.05 \\
0.80 \pm 0.04 \\
0.69 \pm 0.05 \\
0.73 \pm 0.05 \\
0.74 \pm 0.07 \\
0.56 \pm 0.03\end{array}$ & $\begin{array}{l}2.24 \pm 0.14 \\
1.99 \pm 0.10 \\
1.47 \pm 0.06 \\
2.09 \pm 0.19 \\
2.48 \pm 0.31 \\
1.22 \pm 0.13 \\
1.77 \pm 0.18 \\
1.38 \pm 0.17 \\
1.92 \pm 0.15 \\
1.58 \pm C \cdot 22 \\
1.81 \pm 0.18 \\
2.06 \pm 0.24\end{array}$ \\
\hline Mean & & & $774 \pm 100$ & $139 \pm 65$ & $0.68 \pm 0.10$ & $1.83 \pm 0.37$ \\
\hline
\end{tabular}

$\star_{\text {Mean }}$ of 10 observations $( \pm \mathrm{SD})$. 
Table 2 Heart rate responses to the Valsalva manoeuvre in 7 older normal subjects

\begin{tabular}{|c|c|c|c|c|c|c|}
\hline Subject & Age (y) & $\operatorname{sex}$ & Resting $R R$ interval (ms) & Variation in resting $R R$ interval (ms) & Tachycardia ratio* & Valsalva ratio* \\
\hline $\begin{array}{l}13 \\
14 \\
15 \\
16 \\
17 \\
18 \\
19\end{array}$ & $\begin{array}{l}\mathbf{6 6} \\
\mathbf{5 5} \\
\mathbf{5 2} \\
\mathbf{5 6} \\
\mathbf{5 2} \\
\mathbf{6 5} \\
\mathbf{5 7}\end{array}$ & $\begin{array}{l}\mathbf{F} \\
\mathbf{F} \\
\mathbf{F} \\
\mathbf{M} \\
\mathbf{M} \\
\mathbf{M} \\
\mathbf{F}\end{array}$ & $\begin{array}{l}800 \pm 56 \\
884 \pm 44 \\
832 \pm 44 \\
772 \pm 72 \\
800 \pm 80 \\
884 \pm 80 \\
688 \pm 60\end{array}$ & $\begin{array}{r}68 \pm 24 \\
148 \pm 60 \\
72 \pm 28 \\
76 \pm 12 \\
88 \pm 40 \\
92 \pm 24 \\
56 \pm 16\end{array}$ & $\begin{array}{l}0.84 \pm 0.06 \\
0.71 \pm 0.06 \\
0.73 \pm 0.04 \\
0.74 \pm 0.03 \\
0.70 \pm 0.02 \\
0.74 \pm 0.05 \\
0.77 \pm 0.04\end{array}$ & $\begin{array}{l}1.28 \pm 0.15 \\
1.49 \pm 0.16 \\
1.33 \pm 0.08 \\
1.80 \pm 0.14 \\
1.63 \pm 0.08 \\
1.43 \pm 0.14 \\
1.46 \pm 0.11\end{array}$ \\
\hline Mean & 58 & - & $809 \pm 68$ & $86 \pm 30$ & $0.75 \pm 0.05$ & $1 \cdot 49 \pm 0 \cdot 18$ \\
\hline
\end{tabular}

$\star$ Mean of 5 observations $( \pm \mathrm{SD})$.

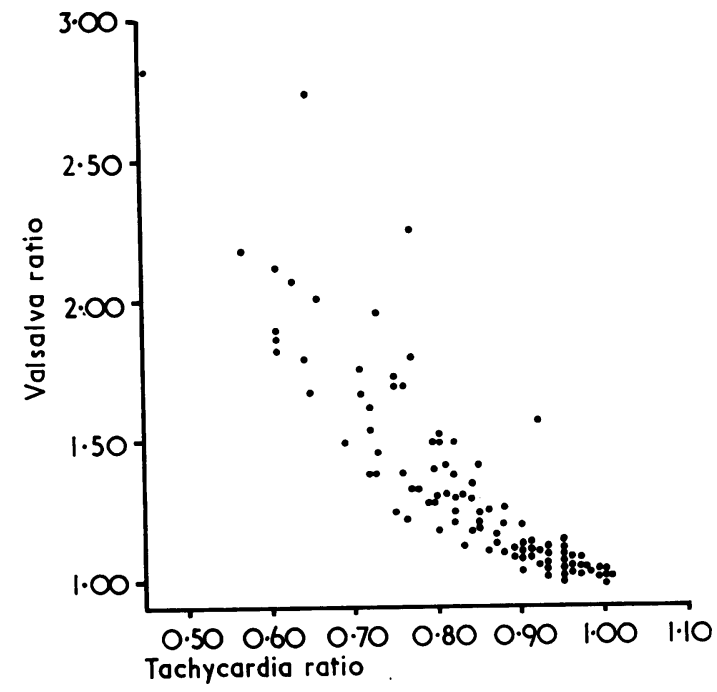

Fig. 1 The tachycardia and Valsalva ratios in 100 diabetics.

Thus, in these 100 diabetics, some of whom had varying degrees of autonomic neuropathy, as determined by an abnormal Valsalva ratio, the variation in resting heart rate was significantly correlated with the results of the Valsalva manoeuvre.

\section{Discussion}

The heart rate response to the Valsalva manoeuvre has often been used to assess cardiac autonomic integrity in diabetes (Nathanielsz and Ross, 1967; Bishnu and Berenyi, 1971; Ewing et al., 1973; Low et al., 1975). In common with other noninvasive methods it has the obvious advantage of being simple, and provided it can be shown to be reproducible, can be used in serial studies as an outpatient procedure. In the only previous observations on the reproducibility of the heart rate response to the Valsalva manoeuvre, Levin (1966) found a difference of less than 10 per cent when observations were re-

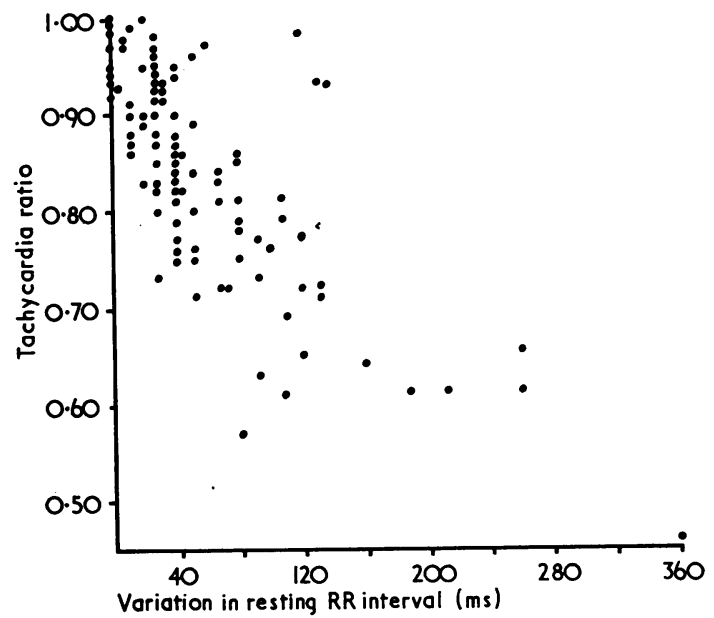

Fig. 2 The relation between the variation in resting $R R$ interval and the tachycardia ratio in 100 diabetics.

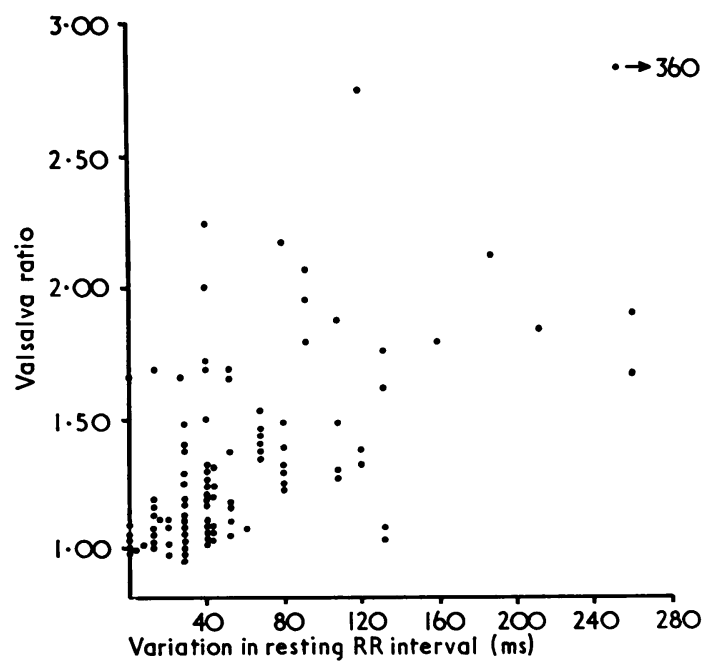

Fig. 3 The relation between the variation in resting $R R$ interval and the Valsalva ratio in 100 diabetics. 
peated after one week; this appears to be a close correlation, but only 2 determinations were made. In the present study we repeated the Valsalva manoeuvre 10 times over a 2-month period in the younger normal subjects and the results show that the heart rate response is indeed reproducible on repeated occasions. This reproducibility can be compared favourably with results obtained using other invasive and noninvasive techniques. It is now possible, within the limits that we have defined, to know whether variations in the response of individuals on repeated occasions are the result of normal variability or of pathological processes. To provide a more valid comparison with the older diabetics, 7 further normal subjects, all over 50 years, repeated the Valsalva manoeuvre 5 times. The reproducibility was slightly better than in the younger normal group, but this may be explained by the shorter period (one week) over which the measurements were made. It is also apparent from these results that variation in heart rate falls slightly with age, as does the Valsalva ratio.

The blood pressure has previously been regarded as the standard method for interpreting the response to the Valsalva manoeuvre though intraarterial cannulation is required. Recently, however, the method of characterising a Valsalva manoeuvre as normal or abnormal by the relation between the percentage increase in diastolic blood pressure and the percentage decrease in pulse pressure during the Valsalva manoeuvre has been shown to be less reliable than originally thought (Johnson and Spalding, 1974). Both Elisberg (1963) and Levin (1966) have shown that the heart rate changes are a reliable indicator of haemodynamic events during and after the strain of the Valsalva manoeuvre. Levin coined the term 'Valsalva ratio', but as can be seen from the study, the 'tachycardia ratio' is closely related to it. Though the tachycardia ratio shows less variation when an individual repeats the Valsalva manoeuvre and therefore appears to be a better index, in practice the Valsalva ratio has the advantage of being independent of the resting heart rate. It has, therefore, been preferred by most investigators.

Heart rate variation measured by either a heart rate meter (Wheeler and Watkins, 1973) or an arrhythmia computer (Murray et al., 1975) has been shown to be abnormal in some diabetics, and is the result of damage to the vagus nerve (Wheeler and Watkins, 1973). In another recent paper (LloydMostyn and Watkins, 1975), diabetics with clinical autonomic neuropathy were subjected to a variety of tests, and found to have both abnormal heart rate variation and abnormal responses to the Valsalva manoeuvre. Details of the responses were not, how- ever, given. The diabetics in our study included those with definite autonomic neuropathy, those with impotence alone, and a group of patients without any symptoms referable to the autonomic nervous system. We thus had a spectrum of diabetics from those with a normal response to the Valsalva manoeuvre to those with no heart rate response at all. It can be seen from Fig. 2 and 3 that a simple measurement of the heart rate variation on the electrocardiogram correlated closely with the Valsalva responses in the group as a whole. Furthermore, only 2 subjects with a Valsalva ratio of 1.20 or less, which we have previously defined as abnormal or borderline (Ewing et al. 1973), had a heart rate variation on the resting electrocardiogram of more than $65 \mathrm{~ms}$. Measurement of the longest and shortest $R R$ intervals from a resting electrocardiogram can thus be used as a simple though not diagnostic screening test for autonomic neuropathy when more sophisticated facilities are not readily available.

We thank Dr. R. J. Prescott for his advice and help in the statistical analysis.

\section{References}

Armitage, P. (1971). Statistical Methods in Medical Research. Blackwell Scientific Publications, Oxford and Edinburgh.

Bishnu, S. K., and Berenyi, M. R. (1971). Circulatory reflex response in diabetic patients with or without peripheral neuropathy. Fournal of the American Geriatrics Society, 19, 159-166.

Elisberg, E. (1963). Heart rate response to the Valsalva maneuver as a test of circulatory integrity. Fournal of the American Medical Association, 186, 200-205.

Ewing, D. J., Campbell, I. W., Burt, A. A., and Clarke, B. F. (1973). Vascular reflexes in diabetic autonomic neuropathy. Lancet, 2, 1354-1356.

Johnson, R. H., and Spalding, J. M. K. (1974). Disorders of the Autonomic Nervous System. Blackwell Scientific Publications, Oxford and Edinburgh.

Levin, A. B. (1966). A simple test of cardiac function based upon the heart rate changes induced by the Valsalva maneuver. American fournal of Cardiology, 18, 90-99.

Lloyd-Mostyn, R. H., and Watkins, P. J. (1975). Defective innervation of heart in diabetic autonomic neuropathy. British Medical fournal, 3, 15-17.

Low, P. A., Walsh, J. C., Huang, C. Y., and McLeod, J. G. (1975). The sympathetic nervous system in diabetic neuropathy. A clinical and pathological study. Brain, 98, 341-356.

Murray, A., Ewing, D. J., Campbell, I. W., Neilson, J. M. M., and Clarke, B. F. (1975). RR interval variations in young male diabetics. British Heart fournal, 37, 882-885.

Nathanielsz, P. W., and Ross, E. J. (1967). Abnormal response to Valsalva maneuver in diabetics-relation to autonomic neuropathy. Diabetes, 16, 462-465.

Wheeler, T., and Watkins, P. J. (1973). Cardiac denervation in diabetes. British Medical fournal, 4, 584-586.

Requests for reprints to Dr. D. J. Ewing, Department of Medicine, Royal Infirmary, Edinburgh EH3 9YW. 